\title{
New Insight into the Dissolution of Epoxy Resin-based Sealers
}

\author{
Inês Ferreira, DMD, MDent, * Stephanie Soares, MSc, ${ }^{\dagger \neq}$ Joana Sousa, DMD, * \\ Joana Barros, DMD, PhD, * Ana Cristina Braga, PhD, Maria Ascensão Lopes, PhD, ${ }^{\star}$ \\ and Irene Pina-Vaz, DMD, $P b D^{*}$
}

\section{Ahstract}

Introduction: The purpose of this study was to evaluate the use of solvents that are not traditionally used in dentistry for the dissolution of an epoxy resin-based sealer and the effect of ultrasonic agitation (UA). Methods: The dissolution of the AH Plus sealer (Dentsply DeTrey, Konstanz, Germany) and the effect of UA in various solvents (eucalyptol, xylene, chloroform, EndoSolv R [Septodont, Cedex, France], EndoSolv E [Septodont], methyl ethyl ketone [MEK], and ethyl acetate) were quantified. The dissolving capacity was assessed by weight loss, Vicker microhardness, scanning electron microscopy (SEM), and X-ray diffraction (XRD). The results were compared with factorial analysis of variance using IBM SPSS Statistics 23.0 software (IBM, Armonk, NY), considering a 0.05 significance level. A preliminary ex vivo study was performed in extracted teeth, with MEK activated by UA as the final irrigation after mechanical removal of the filling material. SEM was used for assessing the cleanliness of the root canal walls. Results: The new solvent proposals, ethyl acetate and MEK, exhibited high dissolution ability, almost reaching chloroform. UA increased dissolution. Vicker values corroborated the dissolution assays. SEM and XRD revealed that solvents affected mainly the organic component of the sealer. Ex vivo results confirmed the immersion model findings. Conclusions: MEK and ethyl acetate proved to be excellent alternatives to chloroform or xylene solvents. MEK presented a high sealer dissolving ability in a short period, especially with UA, without the potential hazards of chloroform, suggesting it is a good approach to AH Plus sealer's dissolution empowered by UA. These results should encourage further studies in order to confirm their clinical relevance. (J Endod 2017;43:1505-1510)

\section{Key Words}

Epoxy resin sealer, microhardness, organic solvents, scanning electron microscopy, solubility
$\mathrm{N}_{\mathrm{r}}^{\mathrm{o}}$ onsurgical endodontic retreatment requires the removal of endodontic filling material to ensure maximum root canal cleaning and bacterial load reduction, focusing on the recovery or maintenance of periapical health (1). However, the complete removal of filling materials, which are often attached to the canal walls and dentinal tubules, is almost impossible $(2,3)$. Although the efficacy of endodontic solvents on gutta-percha is well described, there is some controversy over their action on sealers.

Chloroform, eucalyptol, xylene, EndoSolv R (Septodont, Cedex, France), and EndoSolv E (Septodont) have been used to assist the removal of filling material (4-6). Although chloroform is recognized as one of the most effective solvents (6-8), it is classified as a group 2B carcinogen by the International Agency for Research on Cancer (9).

AH Plus (Dentsply DeTrey, Konstanz, Germany) is a 2-component epoxy resinbased sealer with low solubility (10) and high radiopacity (10) that provides an effective apical seal (11) based on a polymerization reaction of epoxy resin amines. It has been continuously used in comparative studies on the physicochemical, biological, and antimicrobial properties of root canal sealers $(12,13)$. Epoxy resins, the major constituents of $\mathrm{AH}$ Plus, are soluble in oxygenated compounds such as ethyl acetate $\left(\mathrm{CH}_{3}-\mathrm{COO}-\mathrm{CH}_{2} \mathrm{CH}_{3}\right)$ and methyl ethyl ketone (MEK) $\left(\mathrm{CH}_{3}-\mathrm{CO}-\mathrm{CH}_{2}-\mathrm{CH}_{3}\right)$ (14); they are easily obtainable and have a similar cost to those available commercially. MEK and ethyl acetate are colorless volatile liquids soluble in water that are commonly used as industrial solvents (15). The United States Environmental Protection Agency has categorized MEK in group D (not carcinogenic to humans) (16). Ethyl acetate is considered non-mutagenic with low toxicity and the United States Food and Drug Administration has classified it as GRAS (generally regarded as safe) (17).

Passive ultrasonic irrigation has been reported to improve the efficacy of irrigation solutions $(18,19)$, and its use in endodontic solvents has recently been suggested $(4,20)$. The aim of this investigation was to evaluate the dissolution of endodontic epoxy resin-based sealer AH Plus in solvents that are not traditionally used in dentistry, such as ethyl acetate and MEK, and the effect of ultrasonic agitation (UA).

\section{Materials and Methods}

The tested solvents were chloroform (Fisher Scientific UK Ltd, Leicestershire, UK), eucalyptol (Dentaflux, Madrid, Spain), xylene (Sigma-Aldrich, St Louis, MO), EndoSolv

From the *Faculty of Dental Medicine, University of Porto, Porto, Portugal; ${ }^{\dagger}$ REQUIMTE, and Department of Metallurgical Engineering and Materials, Faculty of Engineering, University of Porto, Porto, Portugal; ${ }^{\ddagger}$ CENIMAT/i3N, Department of Materials Science, Nova University of Lisbon, Lisbon, Portugal; and ${ }^{\S}$ ALGORITMI Center, and School of Engineering, University of Minho, Braga, Portugal.

Address requests for reprints to Dr Irene Pina-Vaz, Jardim de Santa Bárbara, n`5, $2^{\circ}$ andar, 4400-314 Braga, Portugal. E-mail address: igapv@sapo.pt 0099-2399/\$ - see front matter

Copyright () 2017 American Association of Endodontists. http://dx.doi.org/10.1016/j.joen.2017.03.015 
R, EndoSolv E, MEK (VWR International SAS, Fontenay-sous-Bois, France), and ethyl acetate (Fisher Scientific UK Ltd). Distilled water was used as a negative control.

Standardized stainless steel molds with a 7-mm diameter and a 3$\mathrm{mm}$ height were cleaned with acetone and fixed on stainless steel blades. AH Plus sealer was prepared following the manufacturers' instructions and placed into the molds. Ten minutes later, the specimens were transferred into a chamber (IKA KS 4000 ic Control; IKA-Werke $\mathrm{GmbH}$, Staufen, Germany) at a constant temperature of $37^{\circ} \mathrm{C}$ for 48 hours. The samples were removed from the molds and weighted on a digital analytical scale before being immersed in the solvents (initial weight, $\mathrm{W}_{0}$ ). At room temperature, the specimens were submerged into each solvent (for 2 and 5 minutes). Afterward, the specimens were removed with tweezers, dipped in $10 \mathrm{~mL}$ distilled water for 10 minutes to neutralize the action of the solvent, blotted dry, and placed again in the chamber at a constant temperature of $37^{\circ} \mathrm{C}$ for 48 hours. The specimens were weighted again (postimmersion weight, $\mathrm{W}_{\mathrm{f}}$ ). The sealer dissolution was quantified as a percentage, considering the difference between the initial and the final weight, according to the following equation:

$$
\text { Dissolution } \%=\frac{W_{0}-W_{f}}{W_{0}} \times 100
$$

The effect of UA was also studied using the protocol described previously; AH Plus specimens $(n=7)$ were immersed in $10 \mathrm{~mL}$ solvent at room temperature and then subjected to agitation in an ultrasonic bath (RETSCH Solutions in Milling \& Sieving, Haan, Germany) to a frequency of $30 \mathrm{kHz}$ for 2 and 5 minutes.

\section{Vickers Microhardness}

Microhardness was measured in fully set sealer samples after immersion for 2 minutes without $\mathrm{UA}$ in the most effective solvents (ie, chloroform, MEK, ethyl acetate, and EndoSolv E). EndoSolv R was also assessed because of its specificity for resin-based sealers. Vickers microhardness was calculated using the durometer Duramin (Struers A/S, Rodovre, Denmark) by applying a load of $10 \mathrm{gf}$ for 15 seconds $(n=7)$. The mean hardness value was calculated after each solvent. The sealer samples not immersed in solvents were used as a control. Softness was considered as any reduction in hardness after exposure to the solvent.

\section{Scanning Electron Microscopy and Energy-dispersive Spectroscopy}

The surface characteristics of the sealer samples were studied using a Quanta 400FEG SEM (FEI, Hillsboro, OR). Previously, the specimens were coated with gold/palladium using an SPI Sputter Coater (SPI Supplies, West Chester, PA) to provide conduction. The examined specimens were sealer samples before (control) and after solvent immersion with and without UA. The solvents used were chloroform and MEK.

\section{X-ray Diffraction Analysis}

$\mathrm{X}$-ray diffraction (XRD) analysis was performed using a Siemens D 5000 diffractometer (D8 Discover; Bruker AXS, Karlsruhe, Germany) with $\mathrm{Cu}-\mathrm{K} \alpha$ radiation $(\lambda=1.5418 \AA$ ). XRD analysis was conducted with a scan range of $25^{\circ}-40^{\circ}(2 \theta)$ using a step size of $0.02^{\circ}$ and a sept time of 2 . Phases were identified using EVA software (Bruker, Coventry, UK). The examined specimens were sealer samples before (control) and after immersion in MEK with and without UA.

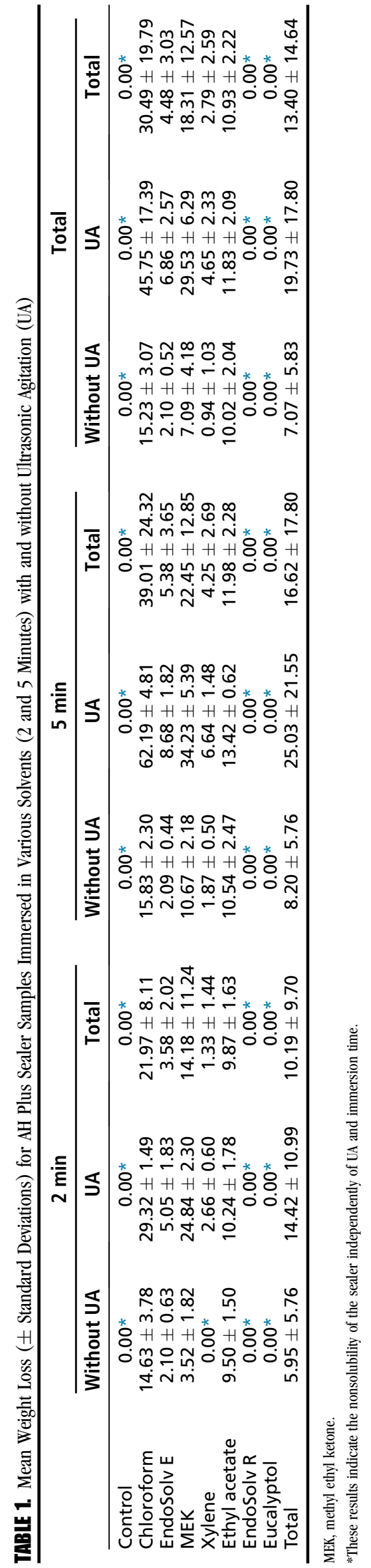


A

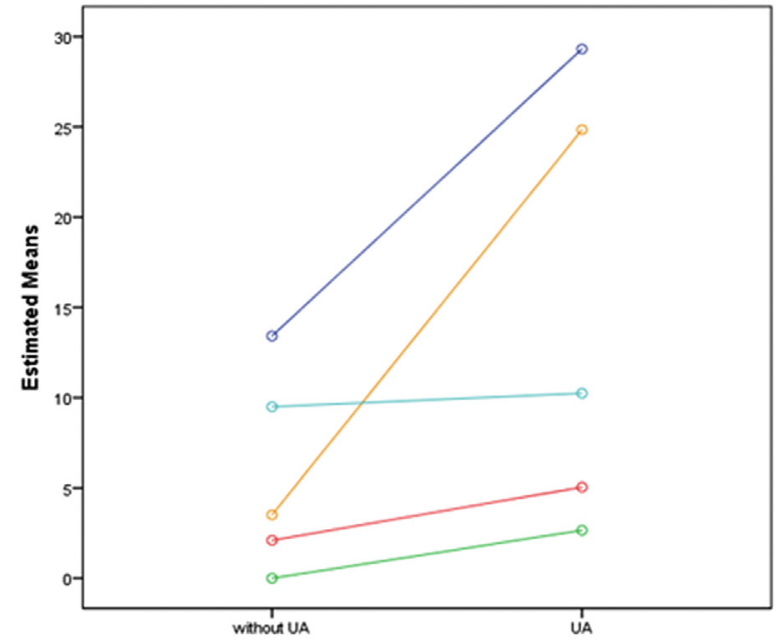

B

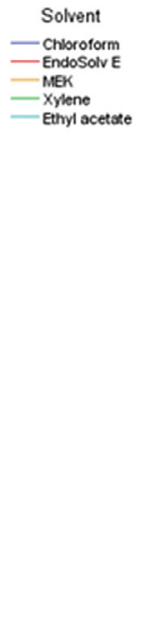

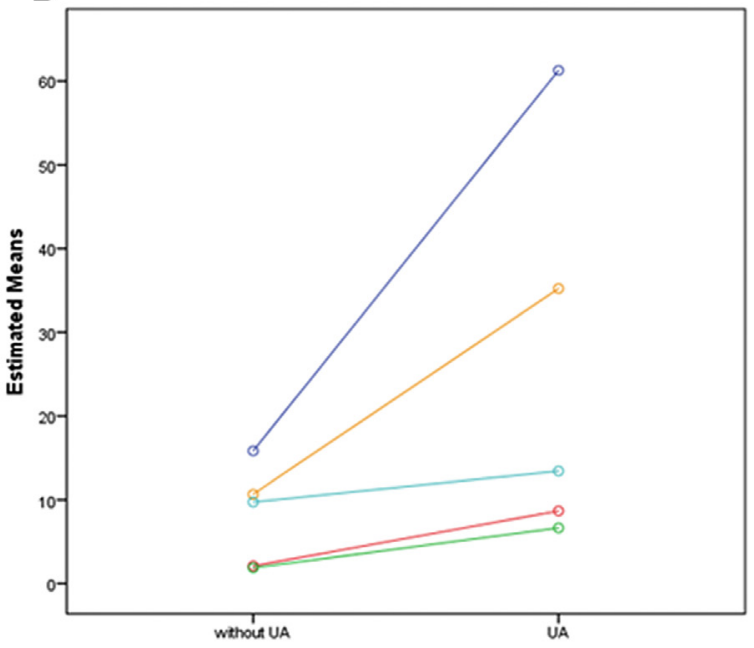

Figure 1. A profile graph for the mean dissolution of the sealer by UA, solvent, and immersion time: (A) 2 minutes and $(B) 5$ minutes.

\section{Statistical Analysis}

Statistical analysis was performed using IBM SPSS Statistics 23.0. software (SPSS Inc, Chicago, IL). Considering the nature of the involved variables, the analysis included the following:

1. Descriptive study, qualitative and quantitative variables (profile graphs and summary statistics tables)

2. Comparative study, factorial analysis of variance (ANOVA) with replication (evaluation of the effect of 3 factors and respective interactions in variables related to the dissolution of AH Plus sealer)

All the conditions for applying the ANOVA procedure were evaluated based on the residuals (normality, zero mean, homogeneity of variance, and independence). Differences were considered to be significant at a value of $P<.05$.

\section{Ex Vivo Test}

Ten human maxillary lateral incisors were prepared with ProTaper Next instruments (Dentsply Maillefer, Ballaigues, Switzerland) up to size X1 using 2.5\% sodium hypochlorite during instrumentation; $1 \mathrm{~mL} \mathrm{17 \%} \mathrm{EDTA} \mathrm{was} \mathrm{used} \mathrm{for} 1$ minute, and a final rinse with $2 \mathrm{~mL} 2.5 \%$ sodium hypochlorite was performed. Teeth were filled with X1 guttapercha cones (Dentsply Maillefer) and AH Plus sealer (single-cone technique). The specimens were stored at $37^{\circ} \mathrm{C}$ and $100 \%$ humidity for 1 week. ProTaper Universal retreatment instruments (Dentsply Maillefer) and Hedstrom files 35 (Dentsply Maillefer) were used for removal

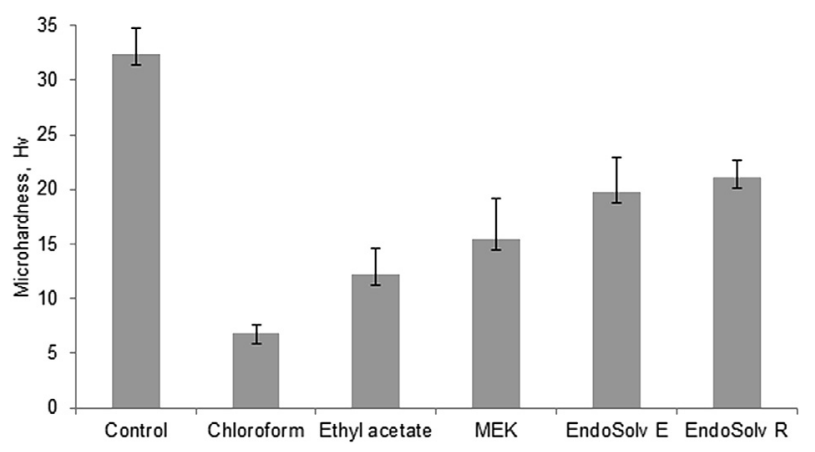

Figure 2. Vickers microhardness mean and standard deviation (control $=$ AH Plus sealer without solvent) in Vickers hardness units (Hv). of the filling material. Specimens were allocated into 2 groups: the control group $(n=5)$ without solvent and the experimental group $(n=5)$ with $1 \mathrm{~mL}$ MEK activated 5 minutes with an IrriSafe 20/21 (Acteon Satelec, Merignac, France) driven by an ultrasonic device (Suprasson P-Max, Acteon Satelec) as the final irrigation.

For the scanning electron microscopic (SEM)/energy-dispersive spectroscopic (EDS) analysis, the teeth were longitudinally cut and coated with gold/palladium using an SPI Sputter Coater (SPI Supplies, West Chester, PA). Backscattered electron images were obtained using a Quanta 400FEG SEM.

\section{Results}

The means and standard deviations of dissolution recorded for the AH Plus sealer samples in the different solvents are summarized in Table 1 . The ANOVA model features $r^{2}=0.98$. As the $P$ values associated with the testing effects in this model are lower than 0.05 , there are statistically significant differences in the mean dissolution of AH Plus sealer samples dependent on the following parameters: immersion time, type of solvent used, UA, solvent-time interaction, agitation-time interaction, solvent-agitation interaction, and solvent-agitation-time interaction.

The results of multiple comparisons between solvents indicate that there are significant differences in the mean values of sealer dissolution, except when comparing xylene with EndoSolv E. The order of the mean dissolution of the sealer in the solvents was as follows:

$$
\mu_{\text {Chloroform }}>\mu_{\text {MEK }}>\mu_{\text {Ethyl acetate }}>\mu_{\text {EndoSolv E }} \cong \mu_{\text {Xylene }}
$$

Regarding UA, its execution caused a significant increase in the mean dissolution of the sealer. The immersion time had an additive effect on the mean dissolution of the sealer; longer time corresponded to increased dissolution. EndoSolv R and eucalyptol presented no efficacy, with and without $\mathrm{UA}$, similar to the control. Figure $1 A$ and $B$ illustrates the effects of factor interaction at 2 and 5 minutes.

\section{Vickers Microhardness}

The hardness values of AH Plus sealer samples before and after immersion in chloroform, ethyl acetate, MEK, EndoSolv E, and EndoSolv $\mathrm{R}$ are reported in Figure 2. The AH Plus sealer's hardness significantly decreased from chloroform, ethyl acetate, MEK, EndoSolv E, and EndoSolv R by $21 \%, 37 \%, 47.8 \%, 61 \%$, and $65.1 \%$, respectively, from the 
original hardness value. No significant differences were found between EndoSolv E and EndoSolv R.

\section{Scanning Electron Microscopy and Energy-dispersive Spectroscopy}

Scanning electron microscopy is a technique that allows the evaluation of the sample's surface topography and its composition depending on the mode used. The secondary electrons mode transmits information about topography, whereas the backscattered electrons mode is used to detect contrast between areas with different chemical compositions. The latter allow differentiating regions rich in light and heavy atoms. The areas rich in lighter atoms (eg, carbon) are darker than the areas rich in atoms with higher atomic numbers (eg, zinc). The SEM/EDS analysis of the AH Plus samples (Fig. $3 A-F$ ) revealed that the dark regions corresponded to the organic phase of the sealer, whereas the bright regions corresponded to the inorganic phase. After immersion in solvents, the organic phase was partly removed, and, therefore, brighter images were acquired from the sealer. An EDS detector was used to perform elemental analysis of these regions, which confirmed that the dark regions were rich in carbon and oxygen, whereas the bright ones were rich in tungstate and calcium.

\section{KRD Analysis}

XRD analysis was performed to identify the crystalline phases in AH Plus before and after being in contact with solvents. A representative pattern obtained from the experiment with MEK (with and without UA) is depicted in Figure 4. The peaks found in the XRD spectra were assigned to calcium tungstate (CaW04 \# 01-077-2233) and zirconium oxide ( $\mathrm{ZrO}_{2}$ \#R040142.1).

The increased intensity of the peaks of AH Plus samples after being in contact with the solvents indicates the presence of a small organic phase. A higher softening ability of the solvent was found to be associated with a higher increase in the intensity of the peaks of XRD patterns.

\section{Ex Vivo Test}

All specimens of the control and experimental groups showed residual filling material (Fig. $5 A-D$ ). In a qualitative analysis, it can be assumed that specimens from the experimental group (MEK + UA) show cleaner root canals walls. The bright areas of the backscattered images correspond to residuals of the sealer as confirmed by the EDS analysis that detects the presence of elements such as zirconium, tungsten, and silicon that are present in the sealer composition.
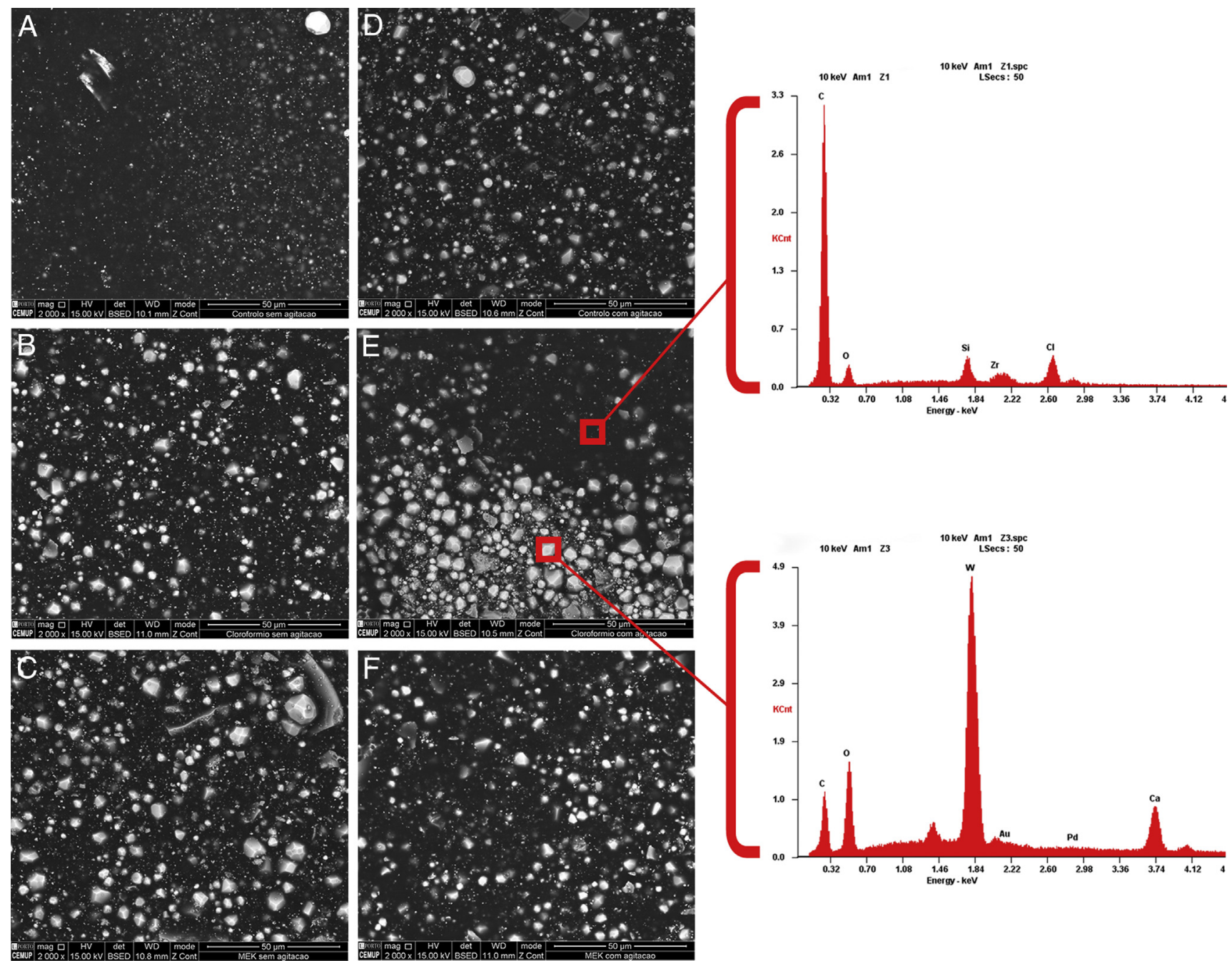

Figure 3. Representative SEM images at $2000 \times$ magnification. Control samples: $(A)$ AH Plus sealer without UA and $(D)$ AH Plus sealer with UA. Experimental groups: $(B)$ chloroform without UA, $(C)$ MEK without UA, $(E)$ chloroform with UA, and $(F)$ MEK with UA and EDS analysis. 


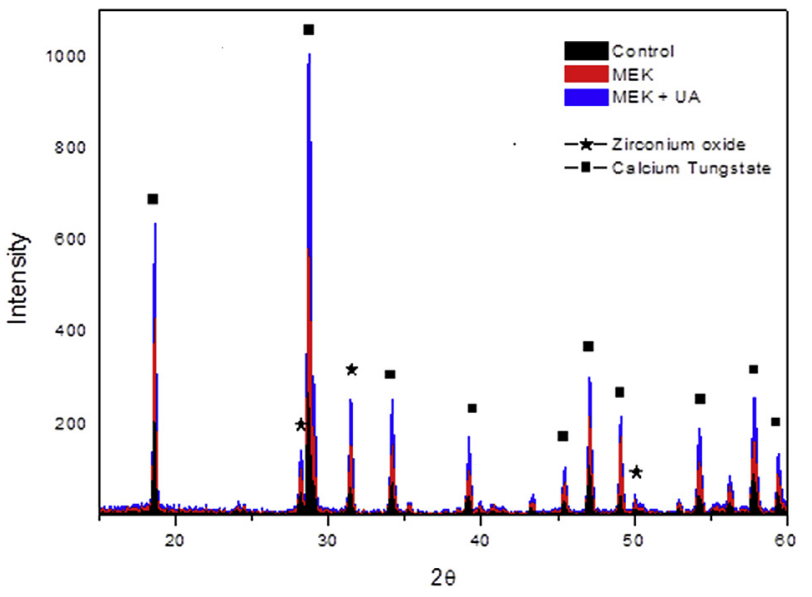

Figure 4. X-ray diffractograms of the sealer after being immersed in MEK (with and without UA) for 2 minutes and the control (sealer samples without immersion).

\section{Discussion}

This investigation revealed that chloroform was the most effective solvent regardless of immersion time and UA execution, thus confirming its efficacy as an endodontic solvent (6-8). Although some authors claim that chloroform can be safe when used in a careful and controlled manner $(21,22)$, there is still some controversy about its use in dentistry. Chloroform carcinogenicity in humans is not clear, but there is sufficient evidence of it in experimental animals (9). These concerns have increased the search for alternatives. Two specific organic solvents for dissolving epoxy resins (ethyl acetate and MEK) were included, showing a good solvent capacity with additional advantages such as exhibiting lower vapor pressure (less volatile), being noncarcinogenic and nonmutagenic, and having low toxicity (23). Thus, their use should be advisable as safer endodontic solvents than chloroform and with similar efficacy.

There are no international standards for analyzing the solubility of root canal filling materials in solvents. However, the ISO has published the 6876:2012 standard (24), which describes the procedure to determine the solubility of set sealer in water. Thus, like in other studies $(7,8)$, the methodology used in the present investigation was an adaptation of that standard.

MEK and ethyl acetate showed the highest ability to dissolve AH Plus sealer after chloroform. EndoSolv E, although not specific for resin sealers, had some solubility effect, as already reported (6). Like in other studies $(6,7,25,26)$, the effect of EndoSolv R, eucalyptol, orange oil, and xylene was null. However, for xylene, 5 minutes of exposure caused some dissolution. On the other hand, it was reported that eucalyptol dissolved AH Plus sealer (27) and that xylene was a good AH Plus solvent $(5,28)$. Longer exposure times might have influenced the results $(7,8)$.

There are few studies about the influence of UA in solvents. In an attempt to simulate the effect of passive ultrasonic irrigation for activating irrigation solutions, the samples were subjected to agitation in an ultrasonic bath to a frequency of $30 \mathrm{kHz}$ in order to mimic a clinical situation (29). The use of chloroform and MEK associated with UA caused a higher dissolution of AH Plus sealer, with MEK presenting similar values to chloroform after 2 minutes of immersion. Also, the effect of xylene was significantly increased by UA, especially at 5 minutes. Alzraikat et al (20) showed that the use of UA significantly increased the efficiency of chloroform. Eucalyptol and EndoSolv R were not influenced by UA; the same inefficacy was also reported $(4,20)$.

The softness of AH Plus sealer was higher in the presence of the new solvent proposals determined by the Vickers microhardness test (ethyl acetate, $12.2 \pm 2.4$ Vickers hardness units [Hv]; MEK, $15.5 \pm 3.7$ $\mathrm{Hv})$, which was only overcome by chloroform $(6.8 \pm 0.8 \mathrm{Hv})$. It seems that there is a correlation between the softness of the sealer after being in
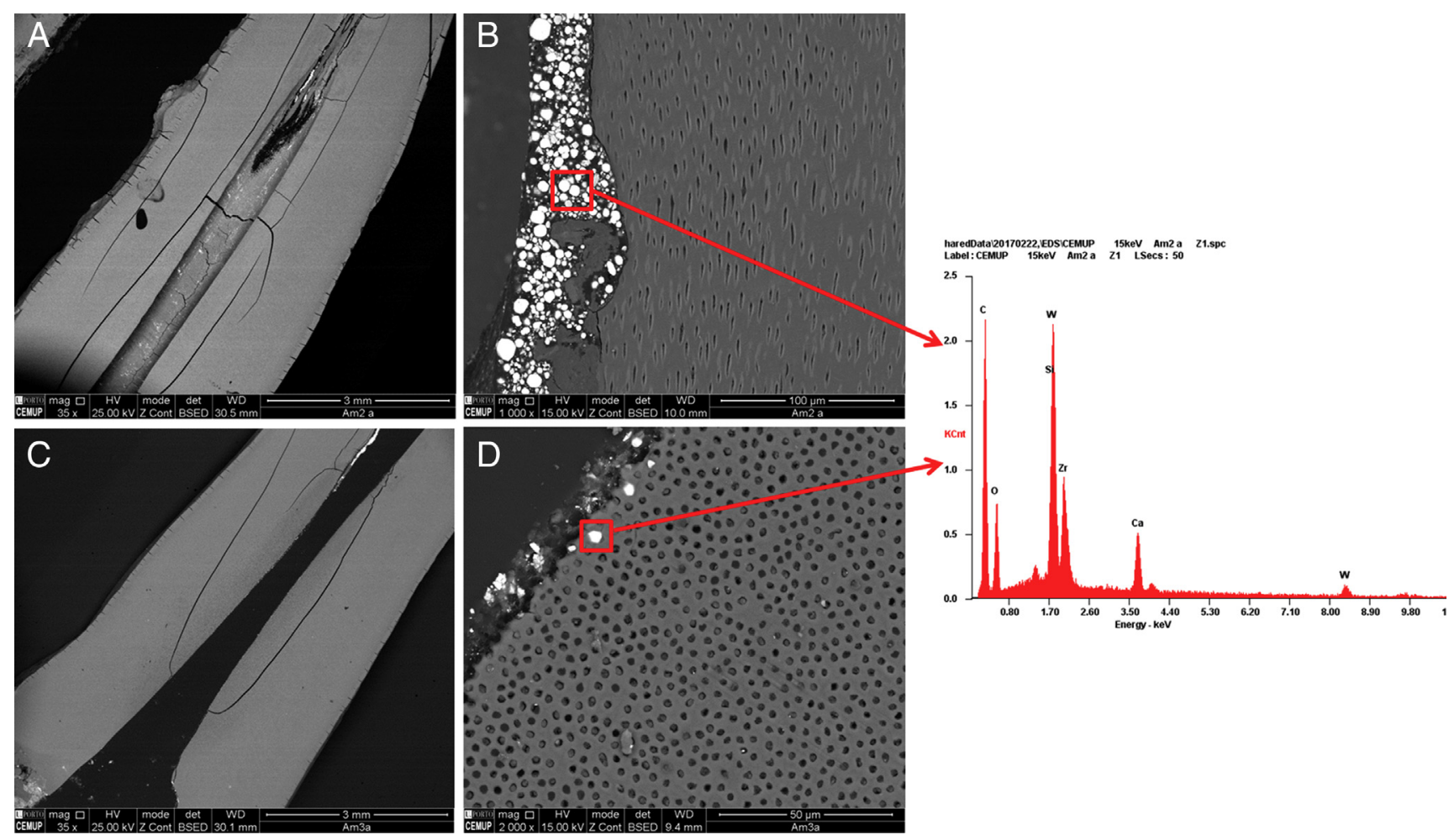

Figure 5. Representative SEM images at different magnifications. Control group: $A$ and $B$. Experimental group: $C$ and $D$ (MEK with UA) and EDS analysis. 
contact with a solvent and its dissolution performance. Regarding EndoSolv R $(21.1 \pm 1.5 \mathrm{Hv})$, although it was not possible to detect any weight loss, there was some softening of the sealer. Similar findings were also reported by Kfir et al (26) regarding chloroform and xylene, with an apparent contradiction between dissolution and hardness. Even with poor dissolution, the authors emphasized the clinical value of sealer softness enabling an easier filling removal with a scrubbing motion (30).

SEM analysis was used to clarify the action of the solvents. The surface topographic changes caused by chloroform and MEK affected mainly the organic component of the AH Plus sealer, either through its dissolution or structural changes, confirmed by XRD analysis.

One of the major drawbacks of the present in vitro study is the extrapolation of the immersion model to clinic because it allows for a full surface contact of sealer with the solvent. In the clinical situation, the sealer is attached to the root canals walls and only partially exposed to the effect of the solvents. Because MEK presented as a new proposal for endodontic solvent from the in vitro studies, the first stage was to assess it in an in vivo approach. In order to evaluate MEK solution in root canal retreatment, a preliminary ex vivo test was performed. The SEM analysis showed promising results with cleaner root canals within the MEK + UA group.

Under the limitations of the present study, MEK and ethyl acetate proved to be excellent alternatives to the traditional chloroform or xylene solvents. MEK presented a high dissolving ability of AH Plus sealer in a short period, especially with UA. Besides almost having reached chloroform's performance, MEK does not have its potential hazards. Thus, the use of MEK can be suggested as a good approach to achieve a better dissolution of AH Plus sealer in the final stages of retreatment procedures. These results should encourage further studies in order to confirm their clinical relevance.

\section{Acknowledgments}

Supported by Fundação para a Ciência e a Tecnologia through the Project UID/EMS/00285/2013.

The authors deny any conflicts of interest related to this study.

\section{References}

1. Duncan HF, Chong BS. Removal of root filling materials. Endod Topics 2008;19:33-57.

2. Kfir A, Tsesis I, Yakirevich E, et al. The efficacy of five techniques for removing root filling material: microscopic versus radiographic evaluation. Int Endod J 2012;45:35-41.

3. Mamootil K, Messer HH. Penetration of dentinal tubules by endodontic sealer cements in extracted teeth and in vivo. Int Endod J 2007;40:873-81.

4. Muller GG, Schonhofen AP, Mora PM, et al. Efficacy of an organic solvent and ultrasound for filling material removal. Braz Oral Res 2013;24:585-90.

5. Martos J, Bassotto AP, Gonzalez-Rodriguez MP, Ferrer-Luque CM. Dissolving efficacy of eucalyptus and orange oil, xylol and chloroform solvents on different root canal sealers. Int Endod J 2011;44:1024-8.

6. Bayram E, Dalat D, Bayram M. Solubility evaluation of different root canal sealing materials. J Contemp Dent Pract 2015;16:96-100.
7. Schafer E, Zandbiglari T. A Comparison of the effectiveness of chloroform and eucalyptus oil in dissolving root canal sealers. Oral Surg Oral Med Oral Pathol Oral Radiol Endod 2002;93:611-6.

8. Whitworth JM, Boursin EM. Dissolution of root canal sealer cements in volatile solvents. Int Endod J 2000;33:19-24.

9. IARC. International Agency for Research on Cancer, Monographs on the Evaluation of Carcinogenic Risk to Humans. Geneva, Switzerland: IARC; 1987: $152-4$.

10. Flores DS, Rached FJ Jr, Versiani MA, et al. Evaluation of physicochemical properties of four root canal sealers. Int Endod J 2011;44:126-35.

11. De Almeida WA, Leonardo MR, Tanomaru Filho M, Silva LA. Evaluation of apical sealing of three endodontic sealers. Int Endod J 2000;33:25-7.

12. Baer J, Maki JS. In vitro evaluation of the antimicrobial effect of three endodontic sealers mixed with amoxicillin. J Endod 2010;36:1170-3.

13. Barros J, Silva MG, Rocas IN, et al. Antibiofilm effects of endodontic sealers containing quaternary ammonium polyethylenimine nanoparticles. J Endod 2014;40:1167-71.

14. Nolte HE. Production of Nanocomposites by Dispersing Nanoparticles in Epoxy Resin Based Polymers. Göttingen, Germany: Sierke-Verlag; 2012.

15. Flick EW. Industrial Solvents Handbook, 5th ed. Westwood, NJ: Noyes Data Corporation; 1998.

16. EPA. Methyl Ethyl Ketone (2-Butanone). Washington, DC: United States Environmental Protection Agency; 2000.

17. EPA. Inert Reassessment - Ethyl Acetate and Amyl Acetate. Washington, DC: United States Environmental Protection Agency; 2006.

18. van der Sluis LW, Versluis M, Wu MK, Wesselink PR. Passive ultrasonic irrigation of the root canal: a review of the literature. Int Endod J 2007;40:415-26.

19. Alves FR, Almeida BM, Neves MA, et al. Disinfecting oval-shaped root canals: effectiveness of different supplementary approaches. J Endod 2011;37:496-501.

20. Alzraikat H, Taha NA, Hassouneh L. Dissolution of a mineral trioxide aggregate sealer in endodontic solvents compared to conventional sealers. Braz Oral Res 2016:30:1-8.

21. Vajrabhaya LO, Suwannawong SK, Kamolroongwarakul R, Pewklieng L. Cytotoxicity evaluation of gutta-percha solvents: chloroform and Gp-Solvent (limonene). Oral Surg Oral Med Oral Pathol Oral Radiol Endod 2004;98:756-9.

22. Chutich MJ, Kaminski EJ, Miller DA, Lautenschlager EP. Risk assessment of the toxicity of solvents of gutta-percha used in endodontic retreatment. J Endod 1998;24:213-6.

23. Sigma-Aldrich. Material safety data sheet. Available at: https://cleanroom.ien.gatech. edu/media/uploads/msds/558.pdf. Accessed May 10, 2016.

24. International Standard ISO 6876: 2012: Dental Root Canal Sealing Materials. Geneva, Switzerland: ISO; 2012:9.

25. Tanomaru Filho M, Jorge EG, Tanomaru JM. Solvent capacity of eucalyptol and xylol on different endodontic sealers. Cienc Odontol Bras 2006;9:60-5.

26. Kfir A, Rosenberg E, Tamse A, Tsesis I. Can epoxy resin-based endodontic sealers be softened within 1-2 nin? An in vitro study with chloroform and xylene. Endod Pract Today 2012;6:189-94.

27. Bodrumlu E, Er 0, Kayaoglu G. Solubility of root canal sealers with different organic solvents. Oral Surg Oral Med Oral Pathol Oral Radiol Endod 2008;106: e67-9.

28. Mushtaq M, Masoodi A, Farooq R, Yaqoob Khan F. The dissolving ability of different organic solvents on three different root canal sealers: in vitro study. Iran Endod J 2012;7:198-202.

29. van der Sluis LW, Vogels MP, Verhaagen B, et al. Study on the influence of refreshment/activation cycles and irrigants on mechanical cleaning efficiency during ultrasonic activation of the irrigant. J Endod 2010;36:737-40.

30. Somma F, Cammarota G, Plotino G, et al. The effectiveness of manual and mechanical instrumentation for the retreatment of three different root canal filling materials. J Endod 2008;34:466-9. 